\title{
The Effects of Antimicrobial Peptide Gel on Angiogenesis and Fibroblast Cells in Periodontal Tissue Regeneration in a Periodontitis Rats Model Exposed by Nicotine
}

\author{
Ika Andriani ${ }^{1,{ }^{*}}$ Atiek Driana Rahmawati ${ }^{2}$ Maulida Nurhasanah ${ }^{3}$ M. Ihza Humanindito ${ }^{3}$
}

\author{
${ }^{1}$ Departement of Periodontia, School of Dentistry, Faculty of Medicine and Health Science, Universitas Muhammadiyah \\ Yogyakarta \\ ${ }^{2}$ Departement of Pedodontia, School of Dentistry, Faculty of Medicine and Health Science, Universitas Muhammadiyah \\ Yogyakarta \\ ${ }^{3}$ School of Dentistry, Faculty of Medicine and Health Science, Universitas Muhammadiyah Yogyakarta \\ *Corresponding author. Email: ikaandriani@yahoo.com
}

\begin{abstract}
Nicotine, a major component of tobacco, is a significant contributing factor for the exacerbation of periodontal diseases. Periodontitis, one of the periodontal diseases, leads to alveolar bone damage triggered by bacteria and exacerbated by nicotine exposure as mostly suffered by smokers. Periodontitis treatment requires an antibacterial for the regeneration process; antimicrobial peptide gel (AMP) has a broad-spectrum antimicrobial that is rarely resistant. This study aims to determine the effect of antimicrobial peptide gel on the regeneration process of periodontitis in rat models exposed to nicotine by observing the number of angiogenesis and fibroblast cells. Twenty-four Wistar rats were divided into four groups based on nicotine exposure, the control group and treated rats. Periodontitis was induced by ligation on the mandibular central incisor for 14 days, and the periodontitis rats were treated with AMP on their sulcus of gingiva on day 1,3, and 7. On day 3, 7, and 14 after treatment, two rats were taken randomly in each group for decapitation, followed by histological processing and examination with Hematoxylin-Eosin. The non-parametric statistic test of Kruskal-Wallis revealed that the value of $\mathrm{p}=0,000$ for angiogenesis and $\mathrm{p}=0,001$ for fibroblast showed significant difference $(\mathrm{p}<0,05)$ between the treated and control groups in both groups with and without exposure to nicotine. Conclusion: The high number of angiogenesis and fibroblast cells in treated rats indicated that the Antimicrobial Peptide Gel successfully accelerated periodontitis' regeneration process in rat models exposed to nicotine.
\end{abstract}

Keywords: angiogenesis, antimicrobial peptide gel, fibroblast, nicotine

\section{INTRODUCTION}

Nicotine is one of the thousands of harmful chemicals present in cigarettes. Of more than 4000 harmful substances contained in cigarettes, including carbon monoxide, hydrogen cyanide, and various other carcinogens, nicotine is the substance with the most concentration in cigarettes. In addition, pharmacologically, this active compound is a significant factor in triggering periodontal disease exacerbation [1].

Nicotine is known to impact periodontal tissues since it can aggravate periodontal diseases, including gingivitis and periodontitis. In the case of gingivitis, it can decrease inflammatory response and may lead to bleeding on probing (BOP). In the case of periodontitis, this substance will increase periodontal tissue damage, pocket depth, adhesions and the risk of tooth loss. This worsening condition is triggered by nicotine, which changes the balance of the gingival biofilm bacteria and the immune system [2]. The immune system, which is divided into the innate immune system and the adaptive immune system, can be influenced by several factors, one of which is the smoking habit. Smoking habit is one of the main factors that can affect the disease's mechanism through decreased immune response, increased oxidative stress, and increased apoptosis cells [3]. Some biofilm bacteria, such as Fusobacterium and P.gingivalis, are found to be higher in smokers. This condition is attributed to the decreasing antibody response in the immune system, one of which is causing the abnormal neutrophil response as shown by an increase in the release of destructive enzymes, which can cause more severe periodontal tissue damage [2]. Nicotine decreases PlateletDerived Growth Factor (PDGF) levels and basic Fibroblast Growth Factor (bFGF), stimulating migration and proliferation of specific cells. As a result, the angiogenesis and wound healing processes are disrupted [4]. Based on the effect of nicotine, which inhibits the inflammatory response to matrix degradation and impedes these leukocytes' performance and mobilization, we can associate the effects of nicotine with the onset and severity of various periodontal diseases [5].

Periodontitis is a chronic inflammatory disease of the periodontal tissue or tooth-supporting tissues, which is caused by the changing body's immune system in handling dental plaque and is exacerbated by biofilm bacteria [6]. Based on the results of the 2017 World Seminar on 
Classification of Periodontal and Peri-Implantitis Diseases and Conditions [7], we can classify periodontitis based on the biological features of the disease and risk factors according to the followings:

Grade A (Slow Rate of Progression), a non-smoking risk factor.

Grade B (Moderate Rate of Progression). The risk factor comes from the smoking of $<10$ cigarettes per day.

Grade C (Rapid Rate of Progression), the risk factor comes from smoking $\geq 10$ cigarettes per day.

The healing process in the periodontal tissue is supported by several cells, including cells that play a role from the inflammatory stage to the final stage, which results in reattachment, including osteoblasts, neutrophils, monocytes, macrophages, lymphocytes, dendrite cells, keratinocytes, and fibroblasts [8]. The initial response is an inflammatory reaction of leukocytes, releasing neutrophils from the gingival blood vessels to the junctional epithelium to attack the bacterial biofilm until it becomes apoptotic tissue [9]. Angiogenesis is an essential element in the healing process of inflammation as it channels oxygen and nutrients so that cells can proliferate adequately [10]. After that, macrophages will contribute through the phagocytosis process to clean apoptotic tissues and activate lymphocytes, which will produce IL-17. Then, fibroblasts take over the role in the remodeling process [9].

The periodontitis healing process in the periodontal tissue is marked by the regeneration of the periodontium structure, which includes reconstituting the alveolar bone, periodontal ligament, and cementum as well as the return of adhesions [11]. Fibroblasts are one of the cells that participate in the reformation of the periodontium tissue structure [12]. Fibroblasts originate from mesenchymal tissue, characterized by the elongated or star-shaped spindles and are very easy to find in connective tissue. In general, fibroblast cells produce various kinds of products, including collagen, proteoglycan, fibronectin, laminin, metalloproteinase, to prostaglandin. Fibroblasts are communicative and adaptive to various surrounding environments, one of which is indicated by the fact that fibroblasts can transform into myofibroblasts [12]. Myofibroblasts contribute to contracting and remodeling the extracellular matrix and forming new tissues [12].

Antimicrobial peptide (AMP) in the oral cavity is the first defense system line against bacterial or microorganism invasion and infection in the oral cavity. The antimicrobial peptide, secreted by the salivary glands, oral epithelial cells and neutrophils, consists of 45 types, all of which are present in the oral cavity to inhibit bacterial and microorganism invasion and infections. Based on this function, the antimicrobial peptide's failure to respond to inflammation will lead to periodontal disease [14]. Different responses and different host conditions that can reduce antimicrobial peptide production can be overcome by administering artificial antimicrobial peptides. JETTINGCLEAN Antimicrobial Peptide Periodontal Gel is an artificial antimicrobial peptide product in the form of a green transparent gel preparation containing an active ingredient, namely antimicrobial peptide RISE-AP12. It contains natural amino acids formulated as a gel that will continuously attack the infected part. The antimicrobial peptide RISE-AP12 is a potent drug, a broad-spectrum, and specific long-term activity for periodontitis caused by a bacterial infection.

The periodontitis healing process's progression is marked by an increase in clinical attachment level (CAL), a reduction in pocket depth, and a radiograph's appearance that shows bone growth. In theory, the principle of healing in periodontal tissues can only be enforced through observational histological studies, which are the only studies to describe the state of tissue recovery [11]. This study's novelty is the treatment of periodontitis in subjects exposed to nicotine with antimicrobials that rarely cause resistance, namely peptide antimicrobials. This study aims to determine the effect of antimicrobial peptide gel on the regeneration of periodontitis in rats exposed to nicotine by observing the number of angiogenesis and fibroblasts cells.

\section{MATERIAL AND METHODS}

This study is an in vivo experimental laboratory carried out at the UGM Integrated Research and Testing Institute (LPPT), UMY Asri Medical Center (AMC) Anatomical Pathology Laboratory, and UMY Molecular Medicine and Therapy (MMT) Laboratory. In this study, 24 male Wistar rats of about 3 months old and weighing about 200-250 grams were divided into two large groups, namely the nicotine exposure group and the non-nicotine exposure group. The nicotine exposure group was provided with $3 \mathrm{mg} / 60 \mathrm{ml}$ nicotine for three consecutive days according to their respective doses based on body weight (16 $\mu \mathrm{gram} / 20$ grams of body weight) by the peritoneal injection process.

On day 4, all groups of rats exposed to or not exposed to nicotine were ligated using 3-0 Silk Ligature thread ligation to induce periodontitis. Prior to ligation, the rats were given a local anesthetic solution in the form of a mixture of Xylazine and Ketamine to minimize pain. $20 \mathrm{mg} / \mathrm{ml}$ Xylazine of $0.5 \mathrm{ml}$ in amount combined with $100 \mathrm{mg} / \mathrm{ml}$ Ketamine of $1 \mathrm{ml}$ was provided to rats according to each rat's respective body weight at a dose of $0.15 \mathrm{ml} / 100$ grams of body weight. The combination of Xylazine and Ketamine could provide an anesthetic effect for 15-30 minutes. Ligation was performed on the gingiva around the mandibular anterior incisor and left for fourteen days to induce inflammation of the rat periodontal tissue.

After fourteen days, the ligation was removed, and various forms of periodontitis were seen in the rats according to the given exposure. After the ligation was removed, decapitation was carried out for each of the rats from the nicotine exposure group and the non-nicotine exposed groups used as the baseline observation. After that, the treatment was immediately carried out according to groups. Group I and II belonged to nicotine exposure groups, while group III and IV were classified as the nonnicotine exposure group. The treatment group consisted of two groups, namely group I, which were exposed to 
nicotine, and group III, which were not exposed to nicotine, each of which was given one smear of JETTINGCLEAN Antimicrobial Peptide Periodontal Gel. The negative control group consisted of two groups, namely group II, which was exposed to nicotine and group IV, which was not exposed to nicotine. Each of them was given distilled water. The treatment was given to the mandibular anterior incisor gingiva of rats which had periodontitis three times a day, namely on day 1,3 , and 7 .

To determine the effect of Antimicrobial Peptide gel on the healing of periodontitis exposed to nicotine, all groups of test animals were decapitated to take their anterior mandibles and make histological specimens. Two rats were decapitated in each group of tested animals on day 3, 7 and 14 after treatment using JETTINGCLEAN Antimicrobial Peptide Periodontal Gel and distilled water. Before decapitation, the rats were anesthetized using $10 \%$ of Ketamine to cause a sedative effect. Then, the rats were decapitated, and their lower jaw of the anterior was cut. The remaining blood and tissue were cleaned using $0.9 \% \mathrm{NaCl}$ solution; then, they were preserved in a labeled container containing $10 \%$ of formaldehyde to create histological preparations. These preparations could observe the rat gingival tissue condition and count the number of angiogenesis and fibroblasts microscopically. Preparations were made at the Anatomical Pathology Laboratory of AMC UMY using Hematoxylin-Eosin (HE) staining.

Histological preparations were calculated by light microscopy with 400x magnification of the mandibular incisors' alveolar crest using six fields of view. The data resulted from the observation of the number of angiogenesis and fibroblasts were tested for normality with an analytical method using the Kolmogorov Smirnov test and a comparison test using the Kruskal Wallis test.

\section{RESULT}

The calculation of the number of neutrophils in all samples in the six visual fields of the periodontal ligament in the alveolar crest area is as follows:

Table 1.1. Number of neutrophils

\begin{tabular}{ccc}
\hline & Nicotine & Non-Nicotine \\
\hline Baseline & $2,17 \pm 0,75$ & $3,17 \pm 1,47$ \\
\hline AMP Day-3 & $2,67 \pm 2,06$ & $0,50 \pm 1,22$ \\
\hline AMP Day-7 & $2,00 \pm 1,78$ & $6,00 \pm 4,51$ \\
\hline AMP Day-14 & $3,00 \pm 2,40$ & $2,67 \pm 1,03$ \\
\hline Aquadest Day-3 & $4,67 \pm 2,42$ & $2,50 \pm 2,51$ \\
\hline Aquadest Day-7 & $1,67 \pm 1,50$ & $3,83 \pm 4,56$ \\
\hline Aquadest Day-14 & $3,17 \pm 2,40$ & $1,50 \pm 1,22$ \\
\hline
\end{tabular}

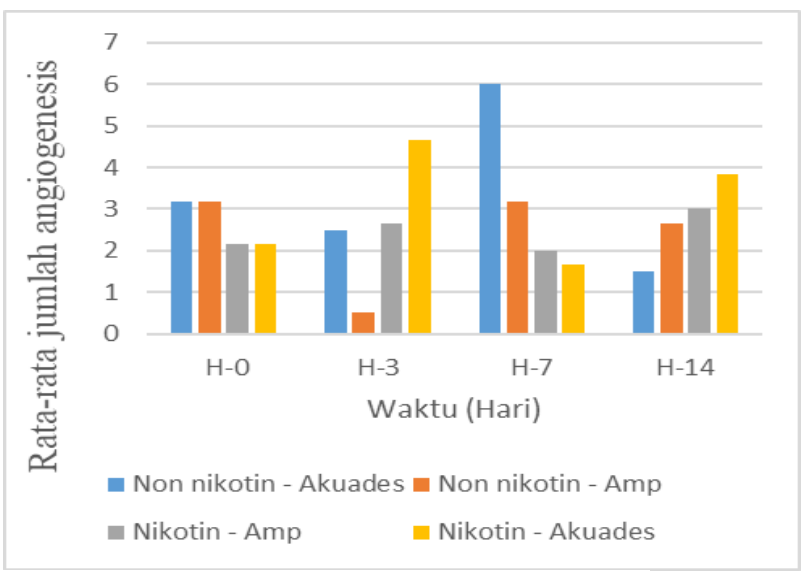

Graph 1. Average Number of Angiogenesis.

The data on the number of angiogenesis are presented in Table 1.1 and Graph 1. The difference in the number of angiogenesis between the exposure and treatment groups was statistically tested using the Kruskal Wallis test.

Table 1.2. Results of the Kr Kruskal Wallis Statistical Test

Angiogenesis

\begin{tabular}{cc} 
& Angiogenesis \\
\hline Chi-Square & 46,148 \\
\hline Df & 14 \\
\hline Asymp. Sig. & 0,000
\end{tabular}

Table 1.2 indicates a significance of $\mathrm{p}=, 000(\mathrm{p}<0.05)$. Thus, it can be concluded that there was a significant difference in the amount of angiogenesis between the exposure and treatment groups.

In addition, the calculation of the number of fibroblasts in sample cells in the six visual fields of the periodontal ligament in the alveolar crest area is as follows:

Table 21. Mean and standard deviation of the number of fibroblasts.

\begin{tabular}{ccc}
\hline & Nicotine & Non-Nicotine \\
\hline Baseline & $29,50 \pm 12,03$ & $34,50 \pm 11,78$ \\
\hline AMP Day-3 & $25,00 \pm 16,69$ & $56,00 \pm 40,16$ \\
\hline AMP Day-7 & $24,50 \pm 8,78$ & $22,67 \pm 7,71$ \\
\hline AMP Day-14 & $40,17 \pm 8,21$ & $71,67 \pm 15,51$ \\
\hline Aquadest Day-3 & $23,33 \pm 12,26$ & $25,17 \pm 5,34$ \\
\hline Aquadest Day-7 & $17,83 \pm 8,38$ & $38,00 \pm 16,72$ \\
\hline Aquadest Day-14 & $30,17 \pm 9,04$ & $23,33 \pm 9,79$ \\
\hline
\end{tabular}




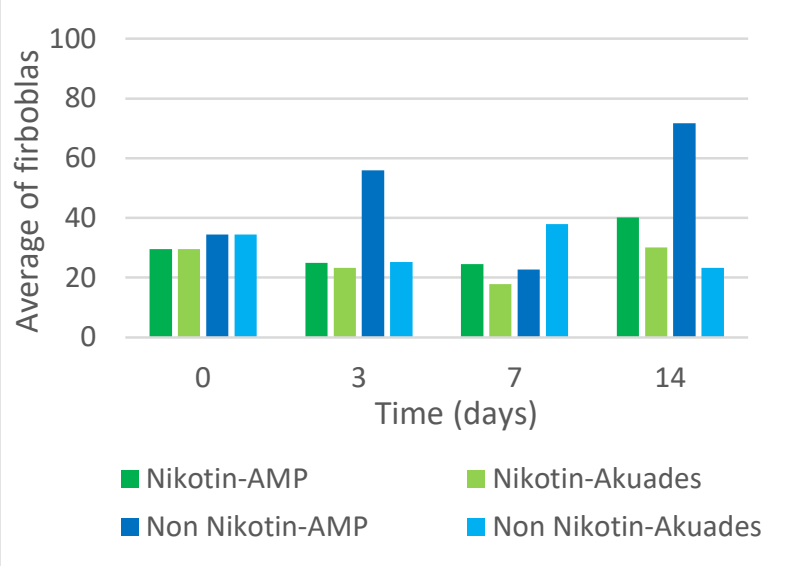

Graph 2. The average number of fibroblasts.

The research data on the number of fibroblasts can be seen in Table 2.1 and Graph 2. Differences number of fibroblasts between exposure and treatment groups were statistically tested using the Kruskal Wallis test.

Table 2.2. Results of Kruskal Wallis Statistical Test

\begin{tabular}{cc}
\hline & Fibroblast \\
\hline Chi-Square & 35.993 \\
\hline Df & 13 \\
\hline Asymp. Sig. & .001
\end{tabular}

Table 2.2 indicates a significance of $\mathrm{p}=0.001$ ( $\mathrm{p}<0.05)$. Thus, it can be concluded that there is a significant

\section{DIFFERENCE IN THE NUMBER OF FIBROBLASTS BETWEEN THE EXPOSURE AND TREATMENT GROUPS. 3. RESULTS}

The statistical tests on the study results revealed the data on baseline subjects indicating that the number of angiogenesis was more common in periodontitis areas of subjects that were not exposed to nicotine. A higher number of fibroblasts was also found in subjects that were not exposed to nicotine than in subjects exposed to nicotine. It indicated that nicotine inhibited the angiogenesis process and automatically slowed down the remodeling process by fibroblasts.

The in vivo studies showed that the angiogenesis process could be affected by exposure to nicotine. It is marked by the fact that nicotine induced for 28 consecutive days could significantly reduce the number of blood vessels. In addition, this substance can also inhibit the expression of angiogenic factors in fibroblasts, lymphocytes and myofibroblasts cells [15].

Other studies had identified that smoking alters important response mechanisms in the homeostasis process, particularly in the remodeling process and prostaglandin E2 (PGE2) production. Cigarettes reduce the activity of PGE2 production as one of the mediators for the differentiation of fibroblasts into myofibroblasts, which contributes to the tissue regeneration process. The reduced activity of fibroblasts in the gingiva will also affect the collagen matrix production, which takes part in the tissue remodeling process [15].

On the 3rd day of treatment, the histological aspect highlighted that the inflammatory response in the form of angiogenesis was less in the treatment with AMP than in the control. Based on the data, the amount of angiogenesis in subjects exposed to nicotine with AMP treatment was lower, which indicated that the effects of nicotine still hampered the angiogenesis process. The previous studies showed that the number of new macrophages on the 3rd day after inflammation would migrate to the inflamed tissue after previously the neutrophils on the 2nd day occupied the inflamed tissue, while the angiogenesis process took place on day 4 to day 21 on a period of cell proliferation in wound tissue $[10,17]$. Other studies stated similar results, in that nicotine could interfere with the angiogenesis process by weakening the ability of endothelial cells to migrate to the wound area and form blood vessels. In addition, nicotine inhibited the angiogenesis process in wound healing by inhibiting angiogenic factors, where the cells that produced these factors, such as lymphocytes, were reduced by nicotine. Reduced lymphocytes could cause decreased ability to form new blood vessels $[18,19]$.

In contrast to the amount of angiogenesis, the histological appearance and data pinpointed an increasing number of fibroblasts in AMP treatment compared to the previous number at baseline. In vitro studies suggested that after neutrophil apoptosis on day 2 to 3, IL-17 would induce fibroblasts' production that would go to the postinflammatory site [9].

On the 7th and 14th days of treatment, the number of angiogenesis and fibroblasts in AMP treatment was higher than the controls in either the group with or without nicotine exposure. It suggested that artificial AMP helped the healing process of periodontitis. This fact was marked by the inflammatory process, which started to normalize based on the number of angiogenesis and continued to the remodeling process as characterized by the increasing number of fibroblasts in AMP treatment compared to that in the control group. This fact is supported by the previous studies that used an AMP dressing application, which highlighted a significant effect on the 7th day as shown by the increasing capillary blood vessel density and the increasing neovascularization ability in tissues. In addition to increasing neovascularization, AMP dressings could also reduce the number of E. Coli and S. Aureus bacteria in the wound tissue well, thereby accelerating the healing process [20]. It is in line with the previous periodontitis healing process theory, which stated that the remodeling phase could be seen after about two weeks as marked by fibroblasts' operation, including through the differentiation of fib into myofibroblasts. These substances would contract and repair the extracellular matrix and were enriched with one type of matrix protein from fibronectin, which helped the cell adhering to collagen. Over time, myofibroblasts will undergo apoptosis and be replaced by new fibroblasts. As a result, it can reduce the secretion of extracellular matrix to avoid excessive scar tissue development. This process can 
last up to a year at the most based on the influence of various internal and external factors [12].

\section{CONCLUSION}

Based on the research and discussion, it is concluded that the antimicrobial peptide gel had an effect on the healing process of periodontitis in nicotine-exposed rats based on the observation of the number of angiogenesis and fibroblasts.

\section{ACKNOWLEDGMENT}

The authors thank the Research, development and community service institutes (LP3M) Universitas Muhammadiyah Yogyakarta

\section{REFERENCES}

[1] Malhotra R, Kapoor A, Grover V, Kaushal S. Nicotine and periodontal tissues. J Indian Soc Periodontol. 2010;14(1):72.

[2] Novak MJ, Novak KF, Preshaw PM. Smoking and Periodontal Disease. In: Carranza's Clinical Periodontology [Internet]. Elsevier; 2012 [cited 2020 Apr 4]. p. 294-301. Retrieved from: https://linkinghub.elsevier.com/retrieve/pii/B978143 7704167000263

[3] Karatas O, Balci Yuce H, Tulu F, Taskan MM, Gevrek F, Toker H. Evaluation of apoptosis and hypoxiarelated factors in gingival tissues of smoker and nonsmoker periodontitis patients. J Periodont Res. 2019 Dec 19;jre.12723.

[4] Ma L, Zheng LW, Sham MH, Cheung LK. Effect of Nicotine on Gene Expression of Angiogenic and Osteogenic Factors in a Rabbit Model of Bone Regeneration. Journal of Oral and Maxillofacial Surgery. 2010 Apr;68(4):777-81.

[5] Ge X, Liu Y-F, Wong Y, Wu L-Z, Tan L, Liu F, et al. Impact of nicotine on the interplay between human periodontal ligament cells and CD4+ T cells. Hum Exp Toxicol. 2016 Sep;35(9):983-90.

[6] Nicu EA, Rijkschroeff P, Wartewig E, Nazmi K, Loos BG. Characterization of oral polymorphonuclear neutrophils in periodontitis patients: a case-control study. BMC Oral Health. 2018 Dec;18(1):149.

[7] Papapanou PN, Sanz M, Buduneli N, Dietrich T, Feres M, Fine DH, et al. Periodontitis: Consensus report of workgroup 2 of the 2017 World Workshop on the Classification of Periodontal and Peri-Implant Diseases and Conditions: Classification and case definitions for periodontitis. J Periodontol. 2018 Jun;89:S173-82.

[8] Chrysanthopoulou A, Mitroulis I, Apostolidou E, Arelaki S, Mikroulis D, Konstantinidis T, et al. Neutrophil extracellular traps promote differentiation and function of fibroblasts: NETs induce fibrosis via differentiation of fibroblasts. J Pathol. 2014 Jul;233(3):294-307.
[9] Cortés-Vieyra R, Rosales C, Uribe-Querol E. Neutrophil Functions in Periodontal Homeostasis. Journal of Immunology Research. 2016;2016:1-9.

[10] DiPietro LA. Angiogenesis and wound repair: when enough is enough. J Leukoc Biol. 2016 Nov;100(5):979-84.

[11]Newman MG. Newman and Carranza's clinical periodontology. 13th edition. Philadelphia, PA: Elsevier, Inc; 2018.

[12] Smith PC, Martínez C, Martínez J, McCulloch CA. Role of Fibroblast Populations in Periodontal Wound Healing and Tissue Remodeling. Front Physiol. 2019 Apr 24;10:270.

[13]Dick MK, Miao JH, Limaiem F. Histology, Fibroblast. In: StatPearls [Internet]. Treasure Island (FL): StatPearls Publishing; 2020 [cited 2020 Apr 6]. Retrieved from: http://www.ncbi.nlm.nih.gov/books/NBK541065/

[14] Gorr S-U, Abdolhosseini M. Antimicrobial peptides and periodontal disease. J Clin Periodontol. 2011 Mar;38 Suppl 11:126-41.

[15] Skinovsky J, Malafaia O, Chibata M, Tsumanuma F, Panegalli Filho F, Martins MVD de C. The influence of nicotine in healing of small bowel anastomoses in rats: angiogenesis and miofibroblasts. Rev Col Bras Cir. 2016 Apr;43(2):87-92.

[16] Romero A, Cáceres M, Arancibia R, Silva D, Couve E, Martínez C, et al. Cigarette smoke condensate inhibits collagen gel contraction and prostaglandin $E$ 2 production in human gingival fibroblasts. J Periodont Res. 2015 Jun;50(3):371-9.

[17] Reinke JM, Sorg H. Wound Repair and Regeneration. Eur Surg Res. 2012;49(1):35-43.

[18] Dhall S, Alamat R, Castro A, Sarker AH, Mao J-H, Chan A, et al. Tobacco toxins deposited on surfaces (third hand smoke) impair wound healing. Clinical Science. 2016 Jul 1;130(14):1269-84.

[19] Abbas OL, Terzi YK, Özatik O, Özatik FY, Turna G, Nar R, et al. Enhancement of vascular endothelial growth factor's angiogenic capacity by the therapeutic modulation of notch signalling improves tram flap survival in rats submitted to nicotine. Journal of Plastic Surgery and Hand Surgery. 2017 Nov 2;51(6):405-13.

[20]Li S, Schmalz G, Schmidt J, Krause F, Haak R, Ziebolz D. Antimicrobial peptides as a possible interlink between periodontal diseases and its risk factors: A systematic review. J Periodont Res. 2018 Apr;53(2):145-55. 